\title{
On Shape Optimization Theory with Fractional Laplacian
}

\author{
Malick Fall ${ }^{1}$, Ibrahima Faye ${ }^{1}$, Alassane $S y^{1}$, Diaraf Seck ${ }^{2}$ \\ ${ }^{1}$ Department of Mathematics, Faculty of Applied Sciences and Information and Communication Technologies, Alioune Diop University, \\ Bambey, Senegal \\ ${ }^{2}$ Department of Mathematics of Decision, Faculty of Economics and Management, Cheikh Anta Diop University, Dakar, Senegal
}

\section{Email address:}

fallm@gmail.com (M. Fall), ibrahima.faye@uadb.edu.sn (I. Faye), alassane.sy@uadb.edu.sn (A. Sy), diaraf.seck@ucad.edu.sn (D. Seck)

\section{To cite this article:}

Malick Fall, Ibrahima Faye, Alassane Sy, Diaraf Seck. On Shape Optimization Theory with Fractional Laplacian. Applied and Computational Mathematics. Vol. 10, No. 3, 2021, pp. 56-68. doi: 10.11648/j.acm.20211003.12

Received: April 17, 2021; Accepted: May 13, 2021; Published: June 26, 2021

\begin{abstract}
The fractional Laplacian is a nonlocal operator that appears in biology, in physic, in fluids dynamic, in financial mathematics and probability. This paper deals with shape optimization problem associated to the fractional laplacian $\Delta^{s}, 0<$ $s<1$. We focus on functional of the form $J(\Omega)=j\left(\Omega, u_{\Omega}\right)$ where $u_{\Omega}$ is solution to the fractional laplacian. A brief review of results related to fractional laplacian and fractional Sobolev spaces are first given. By a variational approach, we show the existence of a weak solution $u_{\Omega}$ belonging to the fractional Sobolev spaces $D^{s, 2}(\Omega)$ of the boundary value problem considered. Then, we study the existence of an optimal shape of the functional $J(\Omega)$ on the class of admissible sets $\mathcal{O}$ under constraints volume. Finally, shape derivative of the functional is established by using Hadamard formula's and an optimality condition is also given.
\end{abstract}

Keywords: Shape Optimisation, Shape Derivative, Optimal Conditions, Fractional Laplacian

\section{Introduction}

In this paper, we are interested for shape optimization problems using fractional laplacian problems. In other words, we look for a domain $\Omega \subset \mathbb{R}^{N}, N \geq 2$ and a function $u_{\Omega}$ solutions to the problem

$$
\inf _{\Omega \subset \mathbb{R}^{N}, \operatorname{vol}(\Omega)=c, \partial \Omega \in \mathcal{C}^{2}} J(\Omega)=j\left(\Omega, u_{\Omega}\right)
$$

where

$$
J(\Omega)=\frac{C(N, 2)}{2} \int_{\mathbb{R}^{N}} \int_{\mathbb{R}^{N}} \frac{\left|u_{\Omega}(x)-u_{\Omega}(y)\right|^{2}}{|x-y|^{N+2 s}} d x d y
$$

and $u_{\Omega}$ is solution to

$$
\left\{\begin{array}{l}
(-\Delta)^{s} u_{\Omega}=f \text { in } \Omega \\
u=0 \text { on } \mathbb{R}^{N} \backslash \Omega .
\end{array}\right.
$$

where $0<s<1, \Omega$ is an open bounded set of $\mathbb{R}^{N}, N \geq 2$.

Shape optimization problems have always interested the research community. A lot of work related to shape optimization is topical today [14], [2], [4], [5], [7], [6]. Allaire and Henrot [2] give a review on recent development in shape optimization. In general, the functional $J$ depends on $\Omega$ and $u_{\Omega}$ solution to a partial differential equation. In most of his papers, the authors consider a domain-dependent functionals with constraint a partial differential equation posed in $\Omega$. In general, the solution $u_{\Omega}$ of this PDE belongs to a Sobolev space. In this paper, we consider a functional $J(\Omega)$ depending on $\Omega$ and $u_{\Omega}$ solution to the fractional Laplacian. Dalibard and Gerad- Varet in [12], showed that it is possible to calculate the shape derivative of the functional considered in the case $s=\frac{1}{2}$. In this work, we try to generalize the results for all $0<s<1$. We have the following the main result.

Theorem 1.1. Let $J(\Omega)$ be a functional given by (2) where $u_{\Omega}$ is solution to (3). Then there exists an open set $\Omega \subset \mathbb{R}^{N}$ of class $\mathcal{C}^{2}$ with $\operatorname{vol}(\Omega)=c$ satisfying

$$
J(\Omega)=\inf _{\omega \subset \mathbb{R}^{N}, \operatorname{vol}(\omega)=c, \partial \omega \in \mathcal{C}^{2}} J(\omega)
$$

Let's consider, a small perturbation of the domain $\Omega$ in the form $\Omega_{t}=\phi_{t}(\Omega)$ where $\phi_{t}$ is a $\mathcal{C}^{1}$ diffeomorphism such that $\phi_{0}=I d$ and $\frac{\partial \phi_{t}}{\partial t}=V$, where $V \in W^{1, \infty}\left(\mathbb{R}^{N}, \mathbb{R}^{N}\right)$. The shape derivative of the function (2) is given by the following 
result.

Theorem 1.2. Let $\Omega \subset \mathbb{R}^{2}$ be an open set of class $\mathcal{C}^{2}$, and
$\Omega_{t}=\phi_{t}(\Omega)$ as below. Then the function $J$ defined in $\Omega_{t}$ by (2) is differentiable and we have

$$
\left(\frac{d J_{f}\left(\Omega_{t}\right)}{d t}\right)_{t=0}=C \lim _{k \longrightarrow \infty} \int_{\mathbb{R}^{2} \times \mathbb{R}^{2}}\left[u_{0}(x) \dot{u}_{0}(y)\left(\frac{\chi_{k}(x+y)+\chi_{k}(x-y)-2 \chi_{k}(x)}{|y|^{2+2 s}}\right)\right] d x d y
$$

where $u_{0}$ is solution to (26) and $\dot{u}_{0}$, the shape derivative of $u_{0}$ is solution to

$$
\left\{\begin{array}{l}
(-\Delta)^{s} \dot{u}_{0}=0 \text { in } \Omega \\
\dot{u}_{0}=0 \text { on } \partial \Omega
\end{array}\right.
$$

The third result is given by

Theorem 1.3. Let $f \in \mathcal{C}^{\infty}\left(\mathbb{R}^{2}\right)$, and let

$$
J_{f}(\Omega)=\inf _{v \in D^{s, 2}(\Omega), v=0 \text { on } \mathbb{R}^{N} \backslash \Omega}\left(<(-\Delta)^{s} v, v>_{D^{s, 2}(\Omega) \times D^{s, 2}(\Omega)}-\int_{\Omega} f v\right) .
$$

Let $V \in \mathcal{C}_{0}^{\infty}\left(\mathbb{R}^{2}\right)$, and $\left(\phi_{t}\right)$ be the flow associated with $V$, namely

$$
\dot{\phi}_{t}=V, \phi_{0}=I d .
$$

Let $\Omega$ be an open set with $\mathcal{C}^{\infty}$ boundary, and let $u_{\Omega, f}$ be the unique minimizer of $J_{f}(\Omega)$, namely.

$$
\left\{\begin{array}{l}
(-\Delta)^{s} u_{\Omega}=f \text { in } \Omega \\
u_{\Omega}=0 \text { on } \mathbb{R}^{N} \backslash \Omega
\end{array}\right.
$$

Then $\partial_{n}^{s} u_{\Omega, f}$ exists, and there exists an explicit constant $k$ such that

$$
\left(\frac{d J_{f}\left(\Omega_{t}\right)}{d t}\right)_{t=0}=k \int_{\partial \Omega}\left(\partial_{n}^{s} u_{\Omega}\right)^{2} V . n d \sigma
$$

More over, the optimal condition is given the following result:

Theorem 1.4. Let $\Omega$ be the solution of the shape optimization problem $\min \left\{J(\Omega, \omega \in \mathcal{O}\}\right.$ under the constraint $u_{\omega}$ solution to (22).

Then, there exists a Lagrange multiplier $\lambda=\lambda(\Omega)$ such that

$$
k\left(\partial_{n}^{s} u_{\Omega}\right)^{2}+\lambda(\Omega)=0,
$$

where $k$ is a constant.

The paper is organized as follows. In section 2, some preliminaries results concerning the fractional Laplacian problem and fractional Sobolev spaces are given. In section 3 , we give the main results of this paper and its proofs: existence result for the shape optimization problem, shape derivative of the functional and optimality condition. In section 4 , we give some concluding remarks and possible extension.

\section{Preliminaries}

In this section, we recall some results that will be useful to in the following of the work.

\subsection{On the Fractional Problem}

Definition 2.1. Let $0<s<1$ and $p \in[1,+\infty[$. We define $W^{s, p}(\Omega)$ as follows

$$
W^{s, p}(\Omega)=\left\{f \in L^{p}(\Omega): \frac{|f(x)-f(y)|}{|x-y|^{\frac{N}{p}+s}} \in L^{p}(\Omega \times \Omega)\right\}
$$

endowed with the usual norm

$$
\|f\|_{W^{s, p}(\Omega)}=\left(\int_{\Omega}|f|^{p} d x+[f]_{W^{s, p}(\Omega)}\right)^{\frac{1}{p}}
$$

where the term

$$
[f]_{W^{s, p}(\Omega)}=\left(\int_{\Omega} \int_{\Omega} \frac{|f(x)-f(y)|^{p}}{|x-y|^{N+p s}} d x d y\right)^{\frac{1}{p}}
$$

is the so-called Gagliardo (semi) norm of $f$.

\subsection{Sobolev Inequalities}

We need the following results whose proof can be found in [15] and [23].

Lemma 2.1. Let $s \in] 0,1[$ and $p \in[1,+\infty[$ such that $s p<$ $N$. Fixe $T>1$, let $N^{\prime} \in \mathbb{Z}$ and $\left(a_{k}\right)_{k}$ a bounded non-negative sequence with $a_{k}=0$ for any $k \geq N^{\prime}$. Then :

$$
\sum_{k \in \mathbb{Z}} a_{k}^{\frac{N-s p}{N}} T^{k} \leq C \sum_{k \in \mathbb{Z}, a_{k} \neq 0} a_{k+1} a_{k}^{\frac{-s p}{N}} T^{k}
$$

with $C=C(N, s, p, T)>0$.

Lemma 2.2. Let $s \in] 0,1[$ and $p \in[1,+\infty[$ such that $s p<$ $N$. Let $f \in L^{\infty}\left(\mathbb{R}^{N}\right)$ with compact support. For any $k \in \mathbb{Z}$ let:

$$
a_{k}:=\left|\left\{|f|>2^{k}\right\}\right|
$$

Then:

$$
\int_{\mathbb{R}^{\mathbb{N}}} \int_{\mathbb{R}^{\mathbb{N}}} \frac{|f(x)-f(y)|^{p}}{|x-y|^{N+p s}} d x d y \geq C \sum_{k \in \mathbb{Z}, a_{k} \neq 0} a_{k+1} a_{k}^{\frac{-s p}{N}} T^{k} .
$$


with $C=C(N, s, p)>0$.

Lemma 2.3. Let $q \in\left[1,+\infty\left[\right.\right.$, let $f: \mathbb{R}^{\mathbb{N}} \longrightarrow \mathbb{R}$ mesurable function. For any $n \in \mathbb{N}$, let:

$$
f_{n}(x):=\max (\min (f(x), n),-n) \forall x \in \mathbb{R}^{\mathbb{N}}
$$

Then:

$$
\lim _{n \longrightarrow+\infty}\left\|f_{n}\right\|_{L^{q}\left(\mathbb{R}^{\mathbb{N}}\right)}=\|f\|_{L^{q}\left(\mathbb{R}^{\mathbb{N}}\right)} .
$$

Theorem 2.1.: Let $s \in] 0,1[$ and $p \in[1,+\infty[$ such that $s p<N$. Then there exists a positive constant $C=C(N, s, p)$ such that, for any mesurable and compactly supported function $f: \mathbb{R}^{N} \longrightarrow \mathbb{R}$, we have

$$
\|f\|_{L^{p *}\left(\mathbb{R}^{N}\right)} \leq C \int_{\mathbb{R}^{N}} \int_{\mathbb{R}^{N}} \frac{|f(x)-f(y)|^{p}}{|x-y|^{N+p s}} d x d y
$$

Where $p *=p *(N, s)$ is the so-called fractional critical exponent and it is equal to $\frac{N p}{N-s p}$. Consequently, the space $W^{s, p}\left(\mathbb{R}^{N}\right)$ is continuously embedded in $L^{q}\left(\mathbb{R}^{N}\right)$ for any $q \in$ $\left[p, p^{*}\right]$.

Proof. If

$$
\int_{\mathbb{R}^{N}} \int_{\mathbb{R}^{N}} \frac{|f(x)-f(y)|^{p}}{|x-y|^{N+p s}} d x d y=+\infty
$$

then nothing to show.

We then assume that

$$
\int_{\mathbb{R}^{N}} \int_{\mathbb{R}^{N}} \frac{|f(x)-f(y)|^{p}}{|x-y|^{N+p s}} d x d y<+\infty
$$

we have two cases: $*$ If $f \in L^{\infty}\left(\mathbb{R}^{N}\right)$ so we set $A_{k}=\{|f|>$ $\left.2^{k}\right\}$ and $a_{k}=\left|A_{k}\right|$, we have:

$$
\begin{aligned}
\|f\|_{L^{p *}\left(\mathbb{R}^{N}\right)}^{p^{*}} & =\sum_{k \in \mathbb{Z}} \int_{A_{k} \backslash A_{k+1}}|f|^{p^{*}} d x \\
\leq & \sum_{k \in \mathbb{Z}} \int_{A_{k} \backslash A_{k+1}}\left(2^{k+1}\right)^{p^{*}} \leq \sum_{k \in \mathbb{Z}}\left(\left(2^{k+1}\right)^{p^{*}} a_{k}\right)
\end{aligned}
$$

because:

$$
\begin{aligned}
A_{k} \backslash A_{k+1} \subset A_{k} & \Longrightarrow\left|A_{k} \backslash A_{k+1}\right| \leq\left|A_{k}\right|=a_{k} \\
& \Longrightarrow\|f\|_{L^{p *}\left(\mathbb{R}^{N}\right)}^{p^{*}} \leq 2^{p}\left(\sum_{k \in \mathbb{Z}} 2^{k p *} a_{k}\right)^{\frac{p}{p^{*}}}
\end{aligned}
$$

with

$$
\frac{p}{p^{*}}=\frac{N-s p}{N}=1-\frac{s p}{N}<1
$$

then we have

$$
\|f\|_{L^{p *}\left(\mathbb{R}^{N}\right)}^{p^{*}} \leq 2^{p} \sum_{k \in \mathbb{Z}} 2^{k p}\left(a_{k}\right)^{\frac{N-s p}{N}}
$$

We set $T=2^{p}$ and we apply the lemma 2.1 , to obtain:

$$
\|f\|_{L^{p *}\left(\mathbb{R}^{N}\right)}^{p^{*}} \leq C \sum_{k \in \mathbb{Z}, a_{k} \neq 0} a_{k+1}\left(a_{k}\right)^{\frac{-s p}{N}} 2^{k p}
$$

with $C=C(N, s, p)$.

Finally according to the lemma 2.2 we have:

$$
\|f\|_{L^{p *\left(\mathbb{R}^{N}\right)}}^{p^{*}} \leq C \int_{\mathbb{R}^{N}} \int_{\mathbb{R}^{N}} \frac{|f(x)-f(y)|^{p}}{|x-y|^{N+p s}} d x d y
$$

with $C=C(N, s, p) *$ If $f \notin L^{\infty}\left(\mathbb{R}^{N}\right)$, we set : $f_{n}:=$ $\max (\min (f(x), n)-n) \forall x \in \mathbb{R}^{N}$. So, the sequence $\left(f_{n}\right)_{n}$ is bounded, and moreover:

$$
\lim _{n \longrightarrow+\infty}\left\|f_{n}\right\|_{L^{q}\left(\mathbb{R}^{\mathbb{N}}\right)}=\|f\|_{L^{q}\left(\mathbb{R}^{\mathbb{N}}\right)} \forall q \in[1,+\infty[.
$$

So according to the first case we have:

$$
\left\|f_{n}\right\|_{L^{p *}\left(\mathbb{R}^{N}\right)}^{p^{*}} \leq C \int_{\mathbb{R}^{N}} \int_{\mathbb{R}^{N}} \frac{\left|f_{n}(x)-f_{n}(y)\right|^{p}}{|x-y|^{N+p s}} d x d y
$$

Moreover:

$$
\lim _{n \longrightarrow+\infty}\left\|f_{n}\right\|_{L^{q}\left(\mathbb{R}^{\mathbb{N}}\right)}=\|f\|_{L^{q}\left(\mathbb{R}^{\mathbb{N}}\right)}
$$

and according to the dominated convergence theorem we have:

$$
\begin{aligned}
& \lim _{n \longrightarrow+\infty} \int_{\mathbb{R}^{N}} \int_{\mathbb{R}^{N}} \frac{\left|f_{n}(x)-f_{n}(y)\right|^{p}}{|x-y|^{N+p s}} d x d y \\
= & \int_{\mathbb{R}^{N}} \int_{\mathbb{R}^{N}} \frac{|f(x)-f(y)|^{p}}{|x-y|^{N+p s}} d x d y
\end{aligned}
$$

which implies:

$$
\|f\|_{L^{p *}\left(\mathbb{R}^{N}\right)}^{p^{*}} \leq C \int_{\mathbb{R}^{N}} \int_{\mathbb{R}^{N}} \frac{|f(x)-f(y)|^{p}}{|x-y|^{N+p s}} d x d y
$$

with $C=C(N, s, p)$.

The following theorems are useful for the proof of the results in the next section. Their proofs are given in [15] and [23].

Theorem 2.2. Let $s \in] 0,1[$ and $p \in[1,+\infty[$ such that $s p<N$. Let $\Omega \subseteq \mathbb{R}^{N}$ be a domain for $W^{s, p}$. Then there exists a positive constant $C=C(N, s, p, \Omega)$ such that for any function $f \in W^{s, p}(\Omega)$ we have

$$
\|f\|_{L^{q}(\Omega)} \leq C\|f\|_{W^{s, p}(\Omega)},
$$

for any $q \in\left[p, p^{*}\right]$; i.e., $W^{s, p}(\Omega)$ is a continuous injection for $L^{q}(\Omega)$ for any $q \in\left[p, p^{*}\right]$.

Theorem 2.3. Let $s \in] 0,1[, p \in[1,+\infty[$ and $q \in[1, p]$. Let $\Omega \subset \mathbb{R}^{N}$ be a bounded extension domain for $W^{s, p}$ and $T$ be a bounded subset of $L^{p}$. Suppose that

$$
\sup _{f \in T}\left(\int_{\Omega} \int_{\Omega} \frac{|f(x)-f(y)|^{p}}{|x-y|^{N+p s}} d x d y\right)<+\infty .
$$

Then $T$ is pre-compact in $L^{q}$.

Corollary 2.1. Let $s \in] 0,1[, p \in[1,+\infty[$ such that $s p<N$. If $q \in\left[1, p^{*}\left[, \Omega \subseteq \mathbb{R}^{N}\right.\right.$ be a bounded extension domain for 
$W^{s, p}$ and $T$ be a bounded subset of $L^{p}$. Suppose that

Then $T$ is pre-compact in $L^{q}$.

$$
\sup _{f \in T}\left(\int_{\Omega} \int_{\Omega} \frac{|f(x)-f(y)|^{p}}{|x-y|^{N+p s}} d x d y\right)<+\infty,
$$

Definition 2.2. Let $f \in S\left(\mathbb{R}^{N}\right) 0<s<1$ and $x \in \mathbb{R}^{N}$, we define

$$
(-\Delta)^{s} f(x)=C(N, s) v_{p}\left(\int_{\mathbb{R}^{N}} \frac{f(x)-f(y)}{|x-y|^{N+2 s}} d y\right)
$$

with

$$
C(N, s)=\frac{4^{s} \Gamma\left(s+\frac{N}{2}\right)}{\pi^{\frac{N}{2}} \Gamma(-s)}
$$

$v_{p}(f)$ is a principal value of $f$.

Lemma 2.4. Let $0<s<1$ and let $(-\Delta)^{s}$ be the fractional Laplacian operator defined by (2.2). Then for any $f \in S\left(\mathbb{R}^{N}\right)$,

$$
(-\Delta)^{s} f(x)=-\frac{1}{2} C(N, s) \int_{\mathbb{R}^{N}} \frac{f(x+y)+f(x-y)-2 f(x)}{|y|^{N+2 s}} d y, \forall x \in \mathbb{R}^{N} .
$$

Lemma 2.5. Let $f \in C_{c}^{\infty}\left(\mathbb{R}^{N}\right)$, we have

$$
\left((x, y) \longmapsto \frac{f(x+y)+f(x-y)-2 f(x)}{|y|^{N+2 s}}\right) \in L^{1}\left(\mathbb{R}^{2 N}\right) .
$$

We define

$$
\mathcal{L}_{s}^{1}=\left\{f: \mathbb{R}^{N} \longrightarrow \mathbb{R}: \int_{\mathbb{R}^{N}} \frac{|f(x)|}{|x-y|^{N+2 s}} d x<+\infty\right\} .
$$

Definition 2.3. Let $\Omega \subset \mathbb{R}^{N}$ be an open set $u \in \mathcal{L}_{s}^{1}$, the distribution $(-\Delta)^{s} u$ is defined by:

$$
<(-\Delta)^{s} u, \varphi>=\int_{\mathbb{R}^{N}} u(-\Delta)^{s} \varphi d x, \forall \varphi \in C_{c}^{\infty}(\Omega) .
$$

Saying that $(-\Delta)^{s} u=f$ in $D^{\prime}(\Omega)$, is equivalent to the very weak formulation

$$
\int_{\mathbb{R}^{N}} u(-\Delta)^{s} \varphi d x=\int_{\Omega} f \varphi d x, \forall \varphi \in C_{c}^{\infty}(\Omega) .
$$

Definition 2.4. We define $D^{s, 2}(\Omega)={\overline{C_{c}^{\infty}(\Omega)}}^{\|\cdot\|_{H^{s}}}$, as the completion of $C_{c}^{\infty}(\Omega)$, which is an Hilbert espace with respect to the norm:

$$
\|\varphi\|_{D^{s, 2}(\Omega)}=\left(\int_{\mathbb{R}^{N}} \int_{\mathbb{R}^{N}} \frac{[\varphi(x)-\varphi(y)]^{2}}{|x-y|^{N+2 s}} d x d y\right)^{\frac{1}{2}} .
$$

If $u \in D^{s, 2}(\Omega) \subset \mathcal{L}_{s}^{1}$ satisfies: $(-\Delta)^{s} u=f$ in $D^{\prime}(\Omega)$, we have the weak formulation:

$$
<(-\Delta)^{s} u, \varphi>_{D^{s, 2}(\Omega)}=\int_{\Omega} f \varphi d x, \forall \varphi \in D(\Omega)
$$

where

$$
<u, \varphi>_{D^{s, 2}(\Omega)}=C(N, s) \int_{\mathbb{R}^{N}} \int_{\mathbb{R}^{N}} \frac{(u(x)-u(y))(\varphi(x)-\varphi(y))}{|x-y|^{N+2 s}} d x d y .
$$

Let $\Omega \subset \mathbb{R}^{N}$ a bounded open set with Lipschitz boundary, and $0<s<1$. Note here for the space of smooth functions with compact support, we take the notation $C_{c}^{\infty}$ instead of $C_{0}^{\infty}$. Consider the bilinear form:

$$
\begin{gathered}
<,>_{D^{s, 2}}: C_{c}^{\infty}(\Omega) \times C_{c}^{\infty}(\Omega) \longrightarrow \mathbb{R} \\
(u, v) \mapsto \int_{\mathbb{R}^{N}} \int_{\mathbb{R}^{N}} \frac{(u(x)-u(y))(v(x)-v(y))}{|x-y|^{N+2 s}} d x d y
\end{gathered}
$$


which is a scaler product on $C_{c}^{\infty}(\Omega)$. We recall that the Hilbert space $D^{s, 2}(\Omega)$ the completion of $C_{c}^{\infty}(\Omega)$.

Lemma 2.6. If $\Omega$ is a bounded Lipschitz open set

$$
D^{s, 2}(\Omega)=\left\{u \in H^{s}\left(\mathbb{R}^{N}\right) \text {, such that } u=0 \text { on } \mathbb{R}^{N} \backslash \Omega\right\} .
$$

Proposition 2.1. Let $0<s<1$ and $\Omega$ a bounded open set subset of $\mathbb{R}^{N}$. Let $f: \Omega \longrightarrow \mathbb{R}$ be a mesurable function with compact support. Then, there exists a positive constant $C=C(N, s, \Omega)$ depending on $N, s$ and $\Omega$ such that

$$
\|f\|_{L^{2}(\Omega)} \leq C\|f\|_{D^{s, 2}(\Omega)} .
$$

Definition 2.5. $O_{e}$ We say that an open set $\Omega$ satisfies the $\epsilon$-cone property if forall $x \in \partial \Omega$, there exists a unit vector $\xi_{x}$, such that $\forall y \in \bar{\Omega} \cap \mathbf{B}(x, \epsilon), C\left(y, \xi_{x}, \epsilon\right) \subset \Omega$.

We denote by $\theta_{\epsilon}$ the following set:

$$
\mathcal{O}_{\epsilon}=\{\Omega \text { ouvert } \Omega \subset D, \Omega \text { a la propriété du } \epsilon-\text { cône }\}
$$

In what the follows, we denote $\mathcal{O}_{\epsilon}$ the set of all open bounded sets $\Omega$ satisfying the $\epsilon$ - cône property. We have also the following compactness result.

Lemma 2.7. : Let $K$ be a compact and $B$ a bounded open of $\mathbb{R}^{N}$. Let $\Omega_{n} \in \mathcal{O}_{\epsilon}$ be a sequence of open sets with $\bar{\Omega}_{n} \subset K \subset$ $B$. Then there is an open $\Omega$ verifying the ownership of the $\epsilon$ -cone and an extracted sequence $\Omega_{n_{k}}$ such as

$$
\begin{aligned}
& \Omega_{n_{k}} \stackrel{H}{\longrightarrow} \Omega, \chi_{\omega_{n_{k}}} \stackrel{L^{1} p \cdot p}{\longrightarrow} \chi_{\Omega}, \\
& \bar{\Omega}_{n_{k}} \stackrel{H}{\longrightarrow}, \partial \Omega_{n_{k}} \stackrel{H}{\longrightarrow} \partial \Omega .
\end{aligned}
$$

\section{Shape Derivative of the Functional}

The objective of this section is to prove that the shape optimization problem (1)-(2) admits a solution $\Omega$, when $u_{\Omega}$ is solution to

$$
\left\{\begin{array}{c}
(-\Delta)^{s} u_{\Omega}=f \text { sur } \Omega \\
u=0 \text { sur } \mathbb{R}^{N} \backslash \Omega .
\end{array}\right.
$$

We get also optimal condition. Before going further, we first prove existence of uniqueness of the solution $u_{\Omega}$ to (3).

\subsection{Existence of Solution to (3)}

These types of problems were first studied by Caffarelli and Sylvestre [9] and references there in, in the case $s=\frac{1}{2}$. The regularity of the solution to this problem is also studied by many authors. Cafarelli et al. [11] prove interior and boundary Schauder regularity estimates depending on the smoothness of the coefficients. Niang [16], proved in his thesis , by using a blow up and compactness analysis, a boundary regularity for the solution to the mixed boundary degenerate elliptic equation. Silvestre in [21] proved also some regularities results of the obstacle problems for a fractionnal power. In this work, we propose to generalize these problems by using a variational approach in the case where $0<s<1$.

$$
\left\{\begin{array}{c}
(-\Delta)^{s} u_{\Omega}=f \operatorname{sur} \Omega \\
u=0 \text { sur } \mathbb{R}^{N} \backslash \Omega .
\end{array}\right.
$$

We begin this section by proving that it exists a solution to (3). We first use the Euler Lagrange equation of (3) in order to transform it into a functional $J(u)$. We have the following theorem

Theorem 3.1. Let $\Omega \subset \mathbb{R}^{N}, N \geq 1$ be an open set of class $\mathcal{C}^{2}$, and $\left.s \in\right] 0,1[$. Then there exist a unique weak solution $u \in D^{s, 2}(\Omega)$ of (3).

In a addition, this solution satisfies the following problem

$$
J(u)=\inf _{v \in D^{s, 2}} J(v, v)
$$

with

$$
J(u, v)=<u, v>_{D^{s, 2}(\Omega)}-\int_{\Omega} f(x) v(x) d x .
$$

Before giving the proof of this theorem, we give the following lemma, which is usefull for the proof.

Lemma 3.1. Let $\left(u_{k}\right)_{k \geq 1} \subset D^{s, 2}(\Omega)$ be a minimizing sequence of (15), i.e.

$$
\lim _{k \longrightarrow+\infty} J\left(u_{k}, u_{k}\right)=\inf _{v \in D^{s, 2}(\Omega)} J(v, v)=m
$$

then $\left(u_{k}\right)_{k \geq 1}$ is bounded in $D^{s, 2}(\Omega)$.

Proof. Let $\left(u_{k}\right)_{k \geq 1} \subset D^{s, 2}(\Omega)$ satisfying (16). Then, there exists $n_{0} \in \mathbb{N}^{*}$ such that for $k \geq n_{0}$,

$$
m \leq J\left(u_{k}, u_{k}\right) \leq m+\frac{1}{k}, \forall k \geq 1 .
$$

From (15) we have by using schwartz inequality's

$$
\int_{\Omega} f u_{k} d x \leq\|f\|_{L^{2}(\Omega)}\left\|u_{k}\right\|_{L^{2}(\Omega)} \leq \frac{1}{2}\|f\|_{L^{2}(\Omega)}^{2}+\frac{1}{2}\left\|u_{k}\right\|_{L^{2}(\Omega)}^{2}
$$

From this last inequality, we get

$$
\Longrightarrow J\left(u_{k}, u_{k}\right) \geq<u_{k}, u_{k}>_{D^{s, 2}(\Omega)}-\frac{1}{2}\|f\|_{L^{2}(\Omega)}^{2}-\frac{1}{2}\left\|u_{k}\right\|_{L^{2}(\Omega)}^{2}
$$


which gives

$$
\left\|u_{k}\right\|_{D^{s, 2}(\Omega)}^{2} \leq J\left(u_{k}, u_{k}\right)+\frac{1}{2}\|f\|_{L^{2}(\Omega)}^{2}+\frac{1}{2}\left\|u_{k}\right\|_{L^{2}(\Omega)}^{2} .
$$

Taking into account the fact that $f \in L^{2}(\Omega)$, the sequence $u_{k} \in L^{2}(\Omega)$ and the functional $J\left(u_{k}, u_{k}\right) \leq m+\frac{1}{k}$, then for $k$ large enough, we show that the quantity on the right hand side of (17) is bounded. Thus, we show that the term in the left hand side of (17) is the norm $\|u\|_{D^{s, k}(\Omega}$ is bounded by a constant which depends only on $f$ and $m$.

Proof. of Theorem 3.1: Multiplying (3) by a test function $v \in D^{s, 2}(\Omega)$ and integrating over $\Omega$ we get

$$
\int_{\Omega}(-\Delta)^{s} u_{\Omega} v d x=\int_{\Omega} f v d x
$$

Then we have

$$
\int_{\mathbb{R}^{N}} \int_{\mathbb{R}^{N}} \frac{\left(u_{\Omega}(x)-u_{\Omega}(y)\right)(v(x)-v(y))}{|x-y|^{N+2 s}} d x d y=\int_{\Omega} f(x) v(x) d x
$$

Let

$$
a(u, v)=\int_{\mathbb{R}^{N}} \int_{\mathbb{R}^{N}} \frac{\left(u_{\Omega}(x)-u_{\Omega}(y)\right)(v(x)-v(y))}{|x-y|^{N+2 s}} d x d y
$$

and

$$
l(v)=\int_{\Omega} f(x) v(x) d x
$$

It is therefore very difficult to find the existence of a solution $u \in D^{s, 2}(\Omega)$ such that $a(u, v)=l(v)$ for all $v \in D^{s, 2}(\Omega)$, using the Lax Milgram theorem, because of the nonlinearity of the term $(-\Delta)^{s}$. To overcome this difficulty, we consider the following functional.

$$
J\left(u_{\Omega}, v\right)=\int_{\mathbb{R}^{N}} \int_{\mathbb{R}^{N}} \frac{\left(u_{\Omega}(x)-u_{\Omega}(y)\right)(v(x)-v(y))}{|x-y|^{N+2 s}} d x d y-\int_{\Omega} f(x) v(x) d x
$$

The objective will be to show that the functional $J$ defined on $D^{s, 2}(\Omega) \times D^{s, 2}(\Omega)$ by (21) is well defined and a solution $u$ of the problem $\min \left\{J\left(u_{\Omega}, u_{\Omega}\right), u_{\Omega} \in D^{s, 2}(\Omega)\right\}$ is a weak solution of (3). For that, let's start by showing the functional $J$ is reduced and does not reach $-\infty$. We have:

$$
\begin{aligned}
& |J(u, v)|=\left|\int_{\mathbb{R}^{2 N}} \frac{(u(x)-u(y))(v(x)-v(y))}{|x-y|^{N+2 s}} d x d y-\int_{\Omega} f(x) v(x) d x\right|>-\infty \\
& |J(u, v)| \leq\left(\int_{\mathbb{R}^{2 N}} \frac{(|u(x)-u(y)|)^{2}}{|x-y|^{N+2 s}}\right)^{\frac{1}{2}}\left(\int_{\mathbb{R}^{2 N}} \frac{\mid(v(x)-v(y) \mid)^{2}}{|x-y|^{N+2 s}}\right)^{\frac{1}{2}}+\int_{\Omega}|f(x) v(x)| \\
& |J(u, v)| \leq\|u\|_{D^{s, 2}(\Omega)}\|v\|_{D^{s, 2}(\Omega)}+\|f\|_{L^{2}(\Omega)}\|v\|_{L^{2}(\Omega)} .
\end{aligned}
$$

We recall that, there exists a constant $C(N, s, \Omega)$ such that $\|u\|_{L^{2}(\Omega)} \leq C(N, s, \Omega)\|u\|_{D^{s, 2}(\Omega)}$

Then we have

$$
\begin{aligned}
& |J(u, v)| \leq\|u\|_{D^{s, 2}(\Omega)}\|v\|_{D^{s, 2}(\Omega)}+\|f\|_{L^{2}(\Omega)}\|v\|_{L^{2}(\Omega)} \\
& |J(u, v)| \leq\|u\|_{D^{s, 2}(\Omega)}\|v\|_{D^{s, 2}(\Omega)}+\left[C^{2}(N, s, \Omega)\|f\|_{D^{s, 2}(\Omega)}\|v\|_{D^{s, 2}(\Omega)}\right]
\end{aligned}
$$

If $u_{\Omega_{k}}=u_{k}$ is a minimizing sequence, then by lemma $3.1, u_{k}$ is bounded in $D^{s, 2}$, there exists a sub-sequence $\left(u_{k_{l}}\right)_{l \geq 1}$ of $\left(u_{k}\right)_{k \geq 1}$ such that

$$
u_{k_{l}} \rightarrow u \in D^{s, 2}(\Omega), u_{k_{l}} \longrightarrow u \in L^{2}(\Omega) \text { and } u_{k_{l}} \rightarrow u \in L^{2}(\Omega) \text {, when } l \longrightarrow \infty .
$$

In consequence:

$$
J\left(u_{k_{l}}, u_{k_{l}}\right)=\int_{\mathbb{R}^{2 N}} \frac{\left|\left(u_{k_{l}}(x)-u_{k_{l}}(y)\right)\right|^{2}}{|x-y|^{N+2 s}} d x d y-\int_{\Omega} f u_{k_{l}} d x \leq m+\epsilon, \quad \forall \epsilon \geq 0 .
$$

Or

$$
\int_{\mathbb{R}^{2 N}} \frac{\left|\left(u_{k_{l}}(x)-u_{k_{l}}(y)\right)\right|^{2}}{|x-y|^{N+2 s}} d x d y \leq \int_{\Omega} f u_{k_{l}} d x+m+\epsilon, \quad \forall \epsilon \geq 0
$$


Passing to the limit in the second member of the above inequality, we get

$$
\int_{\mathbb{R}^{2 N}} \frac{\left|\left(u_{k_{l}}(x)-u_{k_{l}}(y)\right)\right|^{2}}{|x-y|^{N+2 s}} d x d y \leq \int_{\Omega} f u d x+m+\epsilon, \quad \forall \epsilon \geq 0 .
$$

Therefore, passing to the milit, wheh $k \rightarrow+\infty$ we have

$$
\int_{\mathbb{R}^{2 N}} \frac{|u(x)-u(y)|^{2}}{|x-y|^{N+2 s}} d x d y \leq \int_{\Omega} f u d x+m+\epsilon, \quad \forall \epsilon \geq 0 .
$$

From this inequality, we have

$$
J(u, u)=\int_{\mathbb{R}^{2 N}} \frac{|u(x)-u(y)|^{2}}{|x-y|^{N+2 s}} d x d y-\int_{\Omega} f u d x \leq m+\epsilon, \quad \forall \epsilon \geq 0 .
$$

So, we have,

$$
J(u, u) \leq m \Longrightarrow J(u, u)=m .
$$

Then, the functional $J$ admits a minimum $u \in D^{s, 2}(\Omega)$. In the following, we calculate the Frechet derivative of the functional $J(u, u)$. Let $t \in \mathbb{R}$ and $u, v \in D^{s, 2}(\Omega)$ :

$$
\begin{aligned}
& J(u+t v, u+t v)=\frac{1}{2}<u+t v, u+t v>_{D^{s, 2}(\Omega)}-\int_{\Omega} f(u+t v) \\
&=\frac{1}{2}\left(<u, u>_{D^{s, 2}(\Omega)}+2 t<u, v>_{D^{s, 2}(\Omega)}+t^{2}<v, v>_{D^{s, 2}(\Omega)}\right)-\int_{\Omega} f u-t \int_{\Omega} f v d x \\
&=\frac{1}{2}<u, u>_{D^{s, 2}(\Omega)}+t<u, v>_{D^{s, 2}(\Omega)}+\frac{1}{2} t^{2}<v, v>_{D^{s, 2}(\Omega)}-\int_{\Omega} f u d x-t \int_{\Omega} f v d x \\
&=\frac{1}{2}<u, u>_{D^{s, 2}(\Omega)}-\int_{\Omega} f u d x+t<u, v>_{D^{s, 2}(\Omega)}+\frac{1}{2} t^{2}<v, v>_{D^{s, 2}(\Omega)}-t \int_{\Omega} f v d x \\
& J(u+t v, u+t v)=J(u, u)+t<u, v>_{D^{s, 2}(\Omega)}+\frac{1}{2} t^{2}<v, v>_{D^{s, 2}(\Omega)}-t \int_{\Omega} f v d x \\
& \frac{J(u+t v, u+t v)}{t}-J(u, u) \\
& t
\end{aligned}
$$

Taking the limit as $t \rightarrow 0$ we get:

$$
J^{\prime}(u) t=<(-\Delta)^{s} u_{\Omega}-f, v>
$$

Moreover, $J(u)=\min (J(v))$ then $J^{\prime}(u)=0$, then

$$
(-\Delta)^{s} u_{\Omega}-f=0 \Longrightarrow(-\Delta)^{s} u_{\Omega}=f .
$$

\subsection{Existence of Optimal Shape}

In this section we are interested in the existence of an optimal shape $\Omega$, minimizing the functional $J$ defined by (2) according to the set of domain $\mathcal{O}$. Concerning the questions of existence of optimal shape, one can refer to the work of Allaire [1], A. Henrot and M. Pierre [14], Allaire and Henrot [2], Allaire et al. [3], D. Bucur et al. [7], Buttazo et al. [6] and O. Pironneau [17].

In these various works, the authors cited above use various shape functionals. These functionals generally depend on a function $u_{\Omega}$ solution of a certain partial differential equation. In this present work, $u_{\Omega}$ is the solution of a non-fractional partial differential equation. We try to do the same work but in the fractional case using a functional $J(\Omega)$ and $u_{\Omega}$ is a solution of a fractional type equation. Thus we have the following result:

Theorem 3.2. Let $\mathcal{O}=\left\{\omega \subset \mathbb{R}^{N}, \operatorname{vol}(\omega)=c, \partial \omega \in \mathcal{C}^{2}\right\}$, and $J$ defined by (2). Then there exists a domain $\Omega \in \mathcal{O}$ such that

$$
J(\Omega)=\inf _{\omega \in \mathcal{O}} J(\omega)
$$

under the constraints

$$
\left\{\begin{array}{c}
(-\Delta)^{s} u_{\omega}=f \text { sur } \omega \\
u=0 \text { sur } \mathbb{R}^{N} \backslash \omega .
\end{array}\right.
$$

Proof. The functional $J(\Omega)$ defined by (2) is positive because $u_{\Omega}$ i solution of (22)belong to $D^{s, 2}(\Omega)$. It also does not expect the $+\infty$ value. 


$$
\begin{aligned}
0 \leq J(\Omega) & =\frac{C(N, s)}{2} \int_{\mathbb{R}^{2 N}} \frac{(u(x)-u(y))(u(x)-u(y))}{|x-y|^{N+2 s}} d x d y \\
& =\frac{C(N, s)}{2}\|u\|_{D^{s, 2}(\Omega)}<+\infty .
\end{aligned}
$$

Hence $J$ is bounded.

Let $m=\inf _{\Omega \in \mathcal{O}} J(\Omega)$, so there exists a minimizing sequence $\left(\Omega_{n}\right)_{n \in \mathbb{N}} \subset \mathcal{O}$ such that

$$
J\left(\Omega_{n}\right) \longrightarrow m=\inf J(\Omega) .
$$

Since $\Omega_{n} \subset O$, there exists a compact set $K$ such that $\bar{\Omega}_{n} \subset K$. Then according to the compactness lemma 2.7, there is an open set $\Omega$, with $\left|\Omega_{n}\right|=c$ and an extracted sequence $\Omega_{n_{k}}$ such that $\Omega_{n_{k}} \stackrel{H}{\longrightarrow} \Omega$ and $\chi_{\Omega_{n_{k}}} \stackrel{p . p}{\longrightarrow} \chi_{\Omega}$

It remains to show that:

$$
\lim J\left(\Omega_{n_{k}}\right)=J(\Omega)=\inf _{\Omega \in \mathcal{O}_{\epsilon} \text { ou } \mathcal{O}_{a d}} J(\Omega) .
$$

In $\Omega_{n_{k}}, u_{\Omega_{k}}$ is solution to

$$
\left\{\begin{array}{l}
(-\Delta)^{s} u_{\Omega_{n_{k}}}=f \text { in } \Omega_{n_{k}} \\
u_{\Omega_{n_{k}}}=0 \text { in } \mathbb{R}^{N} \backslash \Omega_{n_{k}}
\end{array}\right.
$$

Multiplying (23) by a test function $v=v_{\Omega} \in D^{s, 2}(\Omega)$ and integrating, we get

$$
\int_{\mathbb{R}^{2 N}} \frac{\left(u_{\Omega_{n_{k}}}(x)-u_{\Omega_{n_{k}}}(y)\right)\left(v_{\Omega}(x)-v_{\Omega}(y)\right)}{|x-y|^{N+2 s}} d x d y=\int_{\Omega_{n_{k}}} f(x) v_{\Omega_{n_{k}}}(x) d x \text { for } \forall v \in D^{s, 2}(\Omega) .
$$

And from Lemma 3.1, the sequence $u_{\Omega_{n_{k}}}$ is bounded in $D^{s, 2}\left(\Omega_{n_{k}}\right)$.

Since $\left(u_{\Omega_{n_{k}}}\right)$ is bounded in $D^{s, 2}\left(\Omega_{n_{k}}\right)$, there exists $u_{\Omega}^{*} \in D^{s, 2}(\Omega)$ and an extracted subsequence $\left(u_{\Omega_{n_{k}}}\right)_{k \geq 1}$ of $\left(u_{\Omega_{n_{k}}}\right)$ still denoted by $\left(u_{\Omega_{n_{k}}}\right)_{k \geq 1}$ such that:

$$
\begin{gathered}
\left(u_{\Omega_{n_{k}}}\right)_{k \geq 1} \longrightarrow u_{\Omega}^{*} \in D^{s, 2}(\Omega), \\
\left(u_{\Omega_{n_{k}}}\right)_{k \geq 1} \longrightarrow u_{\Omega}^{*} \in L^{2}(\Omega)
\end{gathered}
$$

and

$$
\left(u_{\Omega_{n_{k}}}\right)_{k \geq 1} \rightarrow u_{\Omega}^{*} \in L^{2}(\Omega) \text {, if } k \longrightarrow \infty .
$$

Passing to the limit when $k \longrightarrow \infty$ and using weak convergence, we get the following formulation

$$
\int_{\mathbb{R}^{2 N}} \frac{\left(u_{\Omega}^{*}(x)-u_{\Omega}^{*}(y)\right)(\varphi(x)-\varphi(y))}{|x-y|^{N+2 s}} d x d y=\int_{\Omega} f(x) \varphi(x) d x, \forall \varphi \in D^{s, 2}(\Omega)
$$

which is the weak formulation of the following problem

$$
\left\{\begin{array}{l}
(-\Delta)^{s} u_{\Omega}^{*}=f \text { in } \Omega \\
u_{\Omega}^{*}=0 \text { in } \mathbb{R}^{N} \backslash \Omega
\end{array}\right.
$$

Finally by taking $\varphi=u_{\Omega_{n_{k}}}$ in (24), we have

$$
\lim \left(\int_{\mathbb{R}^{2 N}} \frac{\left|\left(u_{\Omega_{n_{k}}}(x)-u_{\Omega_{n_{k}}}(y)\right)\right|^{2}}{|x-y|^{N+2 s}} d x d y\right)=\lim \int_{\Omega_{\Omega_{k}}} u_{\Omega_{n_{k}}} f(x)=\int_{\Omega} f(x) u_{\Omega}^{*}=\int_{\mathbb{R}^{2 N}} \frac{\left[u_{\Omega}^{*}(x)-u_{\Omega}^{*}(y)\right]^{2}}{|x-y|^{N+2 s}} d x d y .
$$

In the other hand, we have

$$
\begin{gathered}
\int_{\mathbb{R}^{2 N}} \frac{\left[\left(u_{\Omega_{n_{k}}}(x)-u_{\Omega_{n_{k}}}(y)\right)-\left(u_{\Omega}(x)-u_{\Omega}(y)\right)\right]^{2}}{|x-y|^{N+2 s}}=\int_{\mathbb{R}^{2 N}} \frac{\left[\left(u_{\Omega_{n_{k}}}(x)-u_{\Omega_{n_{k}}}(y)\right)\right]^{2}}{|x-y|^{N+2 s}} \\
-2 \int_{\mathbb{R}^{2 N}} \frac{\left(u_{\Omega_{n_{k}}}(x)-u_{\Omega_{n_{k}}}(y)\right)\left(u_{\Omega}(x)-u_{\Omega}(y)\right)}{|x-y|^{N+2 s}}+\int_{\mathbb{R}^{2 N}} \frac{\left[\left(u_{\Omega}(x)-u_{\Omega}(y)\right)\right]^{2}}{|x-y|^{N+2 s}}
\end{gathered}
$$


Then taking the limits in the right hand side after equality, as $k \longrightarrow \infty$

$$
\lim _{k \longrightarrow \infty}\left(\int_{\mathbb{R}^{2 N}} \frac{\left[\left(u_{\Omega_{n_{k}}}(x)-u_{\Omega_{n_{k}}}(y)\right)\right]^{2}}{|x-y|^{N+2 s}}-2 \int_{\mathbb{R}^{2 N}} \frac{\left(u_{\Omega_{n_{k}}}(x)-u_{\Omega_{n_{k}}}(y)\right)\left(u_{\Omega}(x)-u_{\Omega}(y)\right)}{|x-y|^{N+2 s}}+\int_{\mathbb{R}^{2 N}} \frac{\left[\left(u_{\Omega}(x)-u_{\Omega}(y)\right)\right]^{2}}{|x-y|^{N+2 s}}\right)=0 .
$$

From which have

$$
\begin{aligned}
& \int_{\mathbb{R}^{2 N}} \frac{\left[\left(u_{\Omega_{n_{k}}}(x)-u_{\Omega_{n_{k}}}(y)\right)-\left(u_{\Omega}(x)-u_{\Omega}(y)\right)\right]^{2}}{|x-y|^{N+2 s}}=0 . \\
& \int_{\mathbb{R}^{2 N}} \frac{\left[\left(u_{\Omega_{n_{k}}}(x)-u_{\Omega_{n_{k}}}(y)\right)-\left(u_{\Omega}(x)-u_{\Omega}(y)\right)\right]^{2}}{|x-y|^{N+2 s}}=\int_{\mathbb{R}^{2 N}} \frac{\left(u_{\Omega_{n_{k}}}(x)-u_{\Omega_{n_{k}}}(y)\right)-\left(u_{\Omega}(x)-u_{\Omega}(y)\right)}{|x-y|^{N+2 s}}=0 \\
& \int_{\Omega_{n_{k}}} f\left(u_{\Omega_{n_{k}}}-u_{\Omega}\right)=0
\end{aligned}
$$

Then

so,

$$
u_{\Omega_{n_{k}}}(x)-u_{\Omega_{n_{k}}}(y) \underset{L_{n_{k}} \stackrel{L^{2}}{\longrightarrow}}{\stackrel{L^{2}}{\longrightarrow}} u_{\Omega}(x)-u_{\Omega}(y)
$$

Finally

$$
u_{\Omega_{n_{k}}} \stackrel{D^{s, 2}}{\longrightarrow} u_{\Omega}
$$

$$
\begin{aligned}
\lim _{k \longrightarrow \infty} J\left(\Omega_{n_{k}}\right) & =\frac{C(N, s)}{2} \int_{\mathbb{R}^{N}} \int_{\mathbb{R}^{N}} \frac{\left|u_{\Omega_{n_{k}}}(x)-u_{\Omega_{n_{k}}}(y)\right|^{2}}{|x-y|^{N+2 s}} d x d y \\
& =J(\Omega)=\frac{C(N, s)}{2} \int_{\mathbb{R}^{N}} \int_{\mathbb{R}^{N}} \frac{\left|u_{\Omega}(x)-u_{\Omega}(y)\right|^{2}}{|x-y|^{N+2 s}} d x d y=m
\end{aligned}
$$

We can conclude that there is an open $\Omega^{*}$ which minimizes $J$ and $\Omega^{*} \in \mathcal{O}_{\epsilon}$

\subsection{Shape Derivative}

Let $\Omega$ be a bounded open set of class $\mathcal{C}^{2}$. For $t \geq 0$, let $\Omega_{t}=\phi_{t}(\Omega)$, where for all $t, \phi_{t}$ associated for $V$ is a diffeomorphism of $\mathbb{R}^{N}, N \geq 2$ and satisfies the following properties:

$$
\dot{\phi}_{0}=V,\left|\operatorname{det}\left(\nabla\left(\phi_{t}\right)\right)\right|=j(t, x), \frac{d \phi_{t}}{d t}=-V, \mid \operatorname{det}\left(\nabla \phi_{t}^{-1} \mid=j(-t, x)\right.
$$

For all $V \in \mathcal{C}^{1} \cap W^{1, \infty}\left(\mathbb{R}^{N}\right)$. Let $u_{\Omega_{t}}$ be the solution to the following problem

$$
\left\{\begin{array}{c}
(-\Delta)^{s} u_{\Omega_{t}}=f \text { in } \Omega_{t} \\
u_{\Omega_{t}}=0 \text { in } \mathbb{R}^{N} \backslash \Omega_{t}
\end{array}\right.
$$

Consider also then function (2) defined in $\Omega_{t}$, by

$$
J\left(\Omega_{t}\right)=\frac{C(N, s)}{2} \int_{\mathbb{R}^{N}} \int_{\mathbb{R}^{N}} \frac{\left|u_{\Omega_{t}}(x)-u_{\Omega_{t}}(y)\right|^{2}}{|x-y|^{N+2 s}} d x d y .
$$

where $u_{\Omega_{t}}$ is solution to (26).

In this part we want to calculate the shape derivative of the functional (27). The calculation of shape derivative of the functional requires the knowledge of the shape and the material derivative of the solution $u_{\Omega_{t}}$. In what follows, we recall some definitions and properties usefull for the following

Definition 3.1. We transport the situation on the domain fixed by the change of variables defined by the following transformation : $I d+t V$. We look at the differentiability of $t \longrightarrow u_{t} \circ(I d+t V)$. If this function can be differentiated into $t=0$, we will be able to define the derivative of $u_{t}$ where $\dot{u}(\Omega, V)$ and this derivative is called material derivative.

Definition 3.2. Let $\omega \subset \Omega$ an open fixed ( strictly included in $\Omega$ ). So by definition we have $\omega \subset \Omega_{t}$, for any t small enough. Therefore the function $u_{t}$ is well defined on $\omega$ and it is convenient to look at the limit of the differential quotient:

$$
\lim _{t \rightarrow 0} \frac{u_{t}-u}{t} .
$$


If this limit exists for all $\omega$, we define a function in the whole domain $\Omega$ noted $u^{\prime}$ where $u^{\prime}(\Omega, V)$ and $u^{\prime}$ is called the form derivative of $u$.

We have the following theorem.

Theorem 3.3. Let $\Omega \subset \mathbb{R}^{2}$ be an open set of class $\mathcal{C}^{2}$, and $\Omega_{t}=\phi_{t}(\Omega)$ as below. Then the function $J$ defined by (27) is differentiable and we have

$$
\left(\frac{d J_{f}\left(\Omega_{t}\right)}{d t}\right)_{t=0}=C \lim _{k \rightarrow \infty} \int_{\mathbb{R}^{2} \times \mathbb{R}^{2}}\left[u_{0}(x) \dot{u}_{0}(y)\left(\frac{\chi_{k}(x+y)+\chi_{k}(x-y)-2 \chi_{k}(x)}{|y|^{2+2 s}}\right)\right] d x d y
$$

where $u_{0}$ is solution to (26) and $u_{0}$, the shape derivative of $u_{0}$ is solution to

$$
\left\{\begin{array}{l}
-\Delta^{s} \dot{u}_{0}=0 \text { in } \Omega \\
\dot{u}_{0}=0 \text { on } \partial \Omega
\end{array}\right.
$$

Proof. From the Theorem 3.1, the unique minimizer of the functional

$$
J(u)=\int_{\mathbb{R}^{2}} \int_{\mathbb{R}^{2}} \frac{\left|u_{\Omega}(x)-u_{\Omega}(y)\right|^{2}}{|x-y|^{N+2 s}} d x d y
$$

satisfies the following variational formulas:

$$
<u, v>_{D^{s, 2}(\Omega) \times D^{s, 2}(\Omega)}-\int_{\Omega} f v d x=0 \quad \forall v \in D^{s, 2}(\Omega) .
$$

Then, for $v=u$ we have

$$
\int_{\mathbb{R}^{2}} \int_{\mathbb{R}^{2}} \frac{\left|u_{\Omega}(x)-u_{\Omega}(y)\right|^{2}}{|x-y|^{N+2 s}} d x d y=\int_{\Omega} f v d x
$$

So to study the functional (27) is equivalent to study the functional $J$ defined by

$$
J\left(\Omega_{t}\right)=\int_{\Omega_{t}} f u_{t} d x
$$

Let $v_{t}=u_{t} o \phi_{t}$ then $v_{t}=u_{t} o \phi_{t} \Longrightarrow v_{t} f=u_{t} o \phi_{t} f$ and $v_{t} f o \phi_{t}=u_{t} o \phi_{t} f o \phi_{t} \Longrightarrow v_{t} f o \phi_{t}=u_{t}\left(\phi_{t}\right) f\left(\phi_{t}\right)$.

So, we have $v_{t} f o \phi_{t}=\left(u_{t} f\right)\left(\phi_{t}\right)$ and $\left(u_{t} f\right)\left(\phi_{t}\right)=v_{t} f o \phi_{t}$. Then, the functional becomes

$$
\begin{aligned}
\int_{\Omega} v_{t} f o \phi_{t}(y) j(t, y) d y & =\int_{\Omega}\left(u_{t} f\right)\left(\phi_{t}\right)(y) j(t, y) d y=\int_{\Omega_{t}} f u_{t} \\
J_{f}\left(\Omega_{t}\right) & =\int_{\Omega_{t}} v_{t} f o \phi_{t}(y) j(t, y) d y
\end{aligned}
$$

and the functional $J\left(\Omega_{t}\right)$ becomes

$$
J\left(\Omega_{t}\right)=\int_{\Omega} v_{t} f o \phi_{t}(y)(y) j(t, y) d y
$$

Since $v_{t} \in D^{s, 2}$ is differentiable, then $t \rightarrow J\left(\Omega_{t}\right)$ is differentiable for $t$ in a neiborhood of zero. Using Hadamard formula, we get,

$$
\begin{aligned}
& \left(\frac{d J_{f}\left(\Omega_{t}\right)}{d t}\right)_{t=0}=\int_{\Omega}\left(\dot{v}_{0} f+v_{0}(V . \nabla f+f d i v V)\right) . \\
& \left(\frac{d J_{f}\left(\Omega_{t}\right)}{d t}\right)_{t=0}=\int_{\Omega} v_{0} \operatorname{div}(f V)+\int_{\Omega} \dot{v}_{0} f .
\end{aligned}
$$

For $k \in \mathbb{N}$ large enough, we define $\chi_{k} \in \mathcal{C}_{0}^{\infty}\left(\mathbb{R}^{2}\right)$ by $\chi_{k}(x)=\chi(k r)$, where $\chi \in \mathcal{C}^{\infty}(\mathbb{R})$ and $\chi(\rho)=0$ for $\rho \leq 1, \chi(\rho)=1$ for $\rho \geq 2$. Since $u_{0}=v_{0}$, we have:

$$
\left(\frac{d J_{f}\left(\Omega_{t}\right)}{d t}\right)_{t=0}=\lim _{k \rightarrow \infty}\left(\int_{\mathbb{R}^{2}} \chi_{k} u_{0} d i v(V f)+\frac{d}{d t}\left[\int_{\mathbb{R}^{2}} u_{t}\left(f \chi_{k}\right) o \phi_{t}^{-1} j(-t, .)\right]_{t=0}\right)
$$

For fixed $k$ and for $t$ in a neigbourhood of zero, there exists a compact set $K_{k}$ such that $K_{k} \subset \Omega$ and $\operatorname{supp}_{k} o \phi_{t}^{-1} \subset K_{k}$. 
Since $\dot{u}_{t} \in L_{t}^{\infty}\left(L_{x}^{1}\right)$, we can use the chain rule and write :

$$
\frac{d}{d t}\left[\int_{\mathbb{R}^{2}} u_{t}\left(f \chi_{k}\right) o \phi_{t}^{-1} j(-t, .)\right]_{t=0}=\int_{\mathbb{R}^{2}}\left(u_{0} f \chi_{k}-u_{0} f V . \nabla \chi_{k}-u_{0} \chi_{k} V . \nabla f-u_{0} \chi_{k} f d i v V\right)
$$

we have :

$$
\begin{aligned}
& \left(\frac{d J_{f}\left(\Omega_{t}\right)}{d t}\right)_{t=0}=\lim _{k \longrightarrow \infty} \int_{\mathbb{R}^{2}} \chi_{k} u_{0} \operatorname{div}(V f)+\int_{\mathbb{R}^{2}}\left(u_{0} f \chi_{k}-u_{0} f V . \nabla \chi_{k}-u_{0} \chi_{k} V . \nabla f-u_{0} \chi_{k} f d i v V\right) \\
& \left(\frac{d J_{f}\left(\Omega_{t}\right)}{d t}\right)_{t=0}=\lim _{k \rightarrow \infty} \int_{\mathbb{R}^{2}} \chi_{k} u_{0} \operatorname{div}(V f)+\int_{\mathbb{R}^{2}}-u_{0} V . \nabla \chi_{k}-u_{0} \chi_{k} \operatorname{div} V+\int_{\mathbb{R}^{2}} \dot{u}_{0} f \chi_{k}-\int_{\mathbb{R}^{2}} u_{0} f V . \nabla \chi_{k} \\
& \left(\frac{d J_{f}\left(\Omega_{t}\right)}{d t}\right)_{t=0}=\lim _{k \longrightarrow \infty}\left(\int_{\mathbb{R}^{2}} \chi_{k} u_{0} \operatorname{div}(V f)+\int_{\mathbb{R}^{2}}-u_{0} V . \nabla \chi_{k}-u_{0} \chi_{k} \operatorname{div} V\right)+\lim _{k \longrightarrow \infty}\left(\int_{\mathbb{R}^{2}} u_{0} f \chi_{k}-\int_{\mathbb{R}^{2}} u_{0} f V . \nabla \chi_{k}\right) \\
& \left(\frac{d J_{f}\left(\Omega_{t}\right)}{d t}\right)_{t=0}=\lim _{k \longrightarrow \infty}\left(\int_{\mathbb{R}^{2}} \dot{u}_{0} f \chi_{k}-\int_{\mathbb{R}^{2}} u_{0} f V \cdot \nabla \chi_{k}\right)
\end{aligned}
$$

With the definition of $\chi_{k}$ we can prove that there exists $C>0$ thus that:

$$
\left|\int_{\mathbb{R}^{2}} u_{0} f V \cdot \nabla \chi_{k}\right| \leq \frac{C}{\sqrt{k}}
$$

Such that we have:

$$
\left(\frac{d J_{f}\left(\Omega_{t}\right)}{d t}\right)_{t=0}=\lim _{k \rightarrow \infty}\left(\int_{\mathbb{R}^{2}} i_{0} f \chi_{k}-\int_{\mathbb{R}^{2}} u_{0} f V . \nabla \chi_{k}\right)
$$

Since $(-\Delta)^{s} u_{0}=f$ on the support of $\chi_{k}$, we have :

$$
\int_{\mathbb{R}^{2}} u_{0} f \chi_{k}=\int_{\mathbb{R}^{2}} \dot{u}_{0} \chi_{k}(-\Delta)^{s} u_{0}=\int_{\mathbb{R}^{2}} u_{0}(-\Delta)^{s}\left(\dot{u}_{0} \chi_{k}\right)
$$

Notice also that $\chi_{k}(-\Delta)^{s}\left(\dot{u}_{0}\right)=0$.

Indeed, $\dot{u}_{t}$ is smooth on $K_{k}$ for $t$ small enough. The integral formula makes sense and we have using the laplacian formulas given in Lemma 2.4 :

$$
(-\Delta)^{s} \dot{u}_{0}(x)=C \int_{\mathbb{R}^{2}} \frac{\dot{u_{0}}(x+y)+\dot{u_{0}}(x-y)-2 \dot{u_{0}}(x)}{|y|^{2+2 s}} d y
$$

Then, we have

$$
(-\Delta)^{s} u_{0}=C\left[\frac{d}{d t} \int_{\mathbb{R}^{2}} \frac{u_{t}(x+y)+u_{t}(x-y)-2 u_{t}(x)}{|y|^{2+2 s}}\right]_{t=0} d y=0
$$

we obtain:

$$
\int_{\mathbb{R}^{2}} u_{0} f \chi_{k}=\int_{\mathbb{R}^{2}} u_{0}(-\Delta)^{s} \chi_{k} \dot{u}_{0}
$$

Then:

$$
\left(\frac{d J_{f}\left(\Omega_{t}\right)}{d t}\right)_{t=0}=C \lim _{k \rightarrow \infty} \int_{\mathbb{R}^{2}} u_{0}(-\Delta)^{s} \chi_{k} \dot{u}_{0}
$$

It's follows from Lemma 2.4 that

$$
(-\Delta)^{s} \chi_{k} u_{0}=C \int_{\mathbb{R}^{2}} i_{0}(y)\left(\frac{\chi_{k}(x+y)+\chi_{k}(x-y)-2 \chi_{k}(x)}{|y|^{2+2 s}}\right) d y
$$

From this last formulas, (29) becomes

$$
\left(\frac{d J_{f}\left(\Omega_{t}\right)}{d t}\right)_{t=0}=\lim _{k \longrightarrow \infty}\left(C \int_{\mathbb{R}^{2} \times \mathbb{R}^{2}} u_{0}(x) \dot{u}_{0}(y)\left(\frac{\chi_{k}(x+y)+\chi_{k}(x-y)-2 \chi_{k}(x)}{|y|^{2+2 s}}\right) d x d y\right)
$$

The functional used in our work corresponds to the energy functional relative to the fractional laplacian. In the case of Dirichlet energy, the shape derivative is known, see A. Henrot and M. Pierre [14] and Dalibard and Gerad- Varet [12]. By following the 
proof of the previous theorem, the functional $J$ can be written in the form

$$
J\left(\Omega_{t}\right)=\int_{\Omega_{t}} f u_{t} d x
$$

Considering $v_{t}=u_{t} o \phi_{t}$, we have

$$
\int_{\Omega} v_{t} f o \phi_{t}(y) j(t, y) d y=\int_{\Omega}\left(u_{t} f\right)\left(\phi_{t}\right)(y) j(t, y) d y=\int_{\Omega_{t}} f u_{t}
$$

and the functional $J\left(\Omega_{t}\right)$ becomes

$$
J\left(\Omega_{t}\right)=\int_{\Omega} v_{t} f o \phi_{t}(y)(y) j(t, y) d y
$$

Since $v_{t} \in D^{s, 2}$ is differentiable, then $t \rightarrow J\left(\Omega_{t}\right)$ is differentiable for $t$ in a neiborhood of zero. Using Hadamard formula, we get easily,

$$
\begin{aligned}
& \left(\frac{d J_{f}\left(\Omega_{t}\right)}{d t}\right)_{t=0}=\int_{\Omega}\left(\dot{v}_{0} f+v_{0}(V . \nabla f+f d i v V)\right) . \\
& \left(\frac{d J_{f}\left(\Omega_{t}\right)}{d t}\right)_{t=0}=\int_{\Omega} v_{0} \operatorname{div}(f V)+\int_{\Omega} \dot{v}_{0} f=\int_{\Omega} v_{0} \operatorname{div}(f V)-\int_{\Omega} \dot{v}_{0} \Delta^{s} u_{0}=\int_{\Omega} v_{0} \operatorname{div}(f V)+v_{0} \Delta^{s} \dot{u}_{0}
\end{aligned}
$$

For $s=\frac{1}{2}$, as in the case where the operator is the Laplacian, the shape derivative is known, see $[12,14]$. Using the same approach as in [12], we obtain also an expression of $\frac{d J_{f}\left(\Omega_{t}\right)}{d t}$ in terms on $\frac{\partial^{s} u_{0}}{\partial n}$, i.e.

$$
\left(\frac{d J_{f}\left(\Omega_{t}\right)}{d t}\right)_{t=0}=-\frac{1}{2} \int_{\partial \Omega}(V \cdot n) \frac{\partial^{s} u_{0}}{\partial n} d \sigma,
$$

giving the proof of Theorem 1.3. The idea is to use an approximation of $u_{0}$ and $\dot{u}_{0}$.

\subsection{Optimal Conditions}

In this section, we are interested to an optimal condition. In other words, we look for the relation associated with the optimal condition $\Omega$ and the Lagrange multiplier $\lambda(\Omega)$.

Theorem 3.4. Let $\Omega$ be the solution of the shape optimization problem $\min \left\{J(\Omega, \omega \in \mathcal{O}\}\right.$ under the constraint $u_{\omega}$ solution to (22).

Then, there exists a Lagrange multiplier $\lambda=\lambda(\Omega)$ such that

$$
k\left(\partial_{n}^{s} u_{\Omega}\right)^{2}+\lambda(\Omega)=0
$$

where $k$ is a constant.

Proof. Suppose that $\Omega$ is a minimizer of $J$ under the contraint $|\Omega|=c$, the theorem of Lagrange multipliers then implies that there exists a constant $\lambda$ such that for any group of diffeomorphisms $\left(\phi_{t}\right)_{t \in \mathbb{R}}$,

$$
\frac{d}{d t}\left(J_{f}\left(\phi_{t}\right)+\lambda\left|\phi_{t}\right|\right)=0 \text { for } t=0 .
$$

Assume that $\left(\phi_{t}\right)_{t \in \mathbb{R}}$ is the flow associated with $V \in$ $\mathcal{C}_{0}^{\infty}\left(\mathbb{R}^{2}\right)$. Then we prove that

$$
\left(\frac{d\left|\phi_{t}\left(\Omega_{t}\right)\right|}{d t}\right)_{t=0}=\int_{\partial \Omega} V \cdot n d \sigma .
$$

From (31), we obtain

$$
\left(\frac{d J_{f}\left(\Omega_{t}\right)}{d t}\right)_{t=0}=-\lambda \int_{\partial \Omega} V \cdot n d \sigma .
$$

In an other hand, From Theorem 1.3, we get

$$
\left(\frac{d J_{f}\left(\Omega_{t}\right)}{d t}\right)_{t=0}=k \int_{\partial \Omega}\left(\partial_{n}^{s} u_{\Omega}\right)^{2} V \cdot n d \sigma .
$$

By making the two preceding expressions equal, we have the following optimality conditions

$$
\begin{aligned}
& k \int_{\partial \Omega}\left(\partial_{n}^{s} u_{\Omega}\right)^{2} V \cdot n d \sigma+\lambda \int_{\partial \Omega} V \cdot n d \sigma \\
= & \int_{\partial \Omega}\left[k\left(\partial_{n}^{s} u_{\Omega}\right)^{2}+\lambda\right] V \cdot n d \sigma=0,
\end{aligned}
$$

giving for all $V$,

$$
k\left(\partial_{n}^{s} u_{\Omega}\right)^{2}+\lambda=0 .
$$

Since $V$ is arbitrary, we infer that $\partial_{n}^{s} u_{\Omega}$ is constant on $\Omega$. Moreover, since $u_{\Omega} \geq 0$ on $\Omega$, by maximum principle, $\partial_{n}^{s} u_{\Omega}$ is positive.

\section{Conclusion}

In this work, we have presented a shape optimization problem using a functional $J$ dependent on the domain $\Omega$ and $u_{\Omega}$ solution of the Fractional Laplacian. We have used some usual techniques to show an existence result of optimal shape and we calculate the shape derivative of the problem considered. Finally we found an optimality condition. It would be interesting in future work to consider the same functional under constraint $-\Delta u^{s}+u^{q}=0$ in in $\Omega$, for $0<s<1, q>$ 1. 


\section{References}

[1] G. Allaire, Shape Optimization by Homogenization Method, Springer Verlag, 2001.

[2] G. Allaire and A. Henrot, On some recent Advances in shape optimization, C. R Acad. Sci. Paris, 7742 (01): 338-396, 2001.

[3] G. Allaire, E. Bonnetier, G. Francfort, F. Jouve, Shape optimization by homogenization method, Numerische Mathematik 76 (1997) 27-68.

[4] D. Bucur, A. Henrot, Minimization of the third eigenvalue of the Dirichlet Laplacian, Proc. R. Soc. London 456 (2000) 985-996.

[5] D. Bucur, P. Trebeschi, Shape optimization problem governed by nonlinear state equation. Proc. Roy. Soc. Edinburgh, 128 A (1998), 945-963.

[6] G. Buttazo, G. Dalmazo, An existence for a class of shape optimization problems. Arch. Rational Mech. Anal. 122 (1993) 183-195

[7] D. Bucur, J. P. Zolésio, Dimensional Shape Optimization under Capacity Constraints. J. Differential Equations, 123 (2) (1995), 504-522.

[8] G. Buttazzo, P. Trebeschi, The role of monotonicity in some shape optimization problems. Calculus of variations and differential equations (Haifa, 1998), 41-55, Chapman- Hall / CRC Res. Notes Math., 410, ChapmanHall/CRD, Boca Raton, FL, 2000.

[9] L. Caffarelli, L. Silvestre, An extension problem related to the fractional Laplacian. Comm. P. D. E. 32 (8), 12451260 (2007).

[10] L. Caffarelli, L. Silvestre An Extension Problem Related to the Fractionnal Laplacian An Extension Problem Related to the Fractionnal Laplacian, Communications in Partial Differential Equation: 37-41, 2007.

[11] L. A. Cafarelli and P. R. Stinga, Fractional elliptic equations, Caccioppoli estimates and regularity, arXiv: 1409. $7721 \mathrm{v} 2,27$ janvier 2015.

[12] A. L. Dalibard and D. Gerard-Varet,On Shape optimization problems involving the fractionnal laplacian, arXiv: 1202.4920 v1 [marh.Ap] 22 Feb 2012.

[13] G. Guy and S. Osher, Nonlocal operators with applications to image processing, multiscale model. Simul. 7 (2008), no.3, 1005-1028. MR 2480109.

[14] A. Henrot, M. Pierre, Shape Variation and Optimization, EMS Tracts in Mathematics Vol. 28, 2018.

[15] E. D. I. Nezza, G. Palatucci, and Enrico valdinoci, Hitchhiker's guide to the fractionnal sobolev spaces, BULL. Sci. MATH. 2012; 136 : 521-573.

[16] A. Niang, Regularity of Solutions to Elliptic Partial Differential Equations with Mixed Boundary Conditions, Phd thesis, UCAD of Dakar, 2020.

[17] O. Pironneau, Optimal Shape Design for Elliptic Systems, Springer Verlag, New York, 1984.

[18] V. Schulz, M. Siebenborn and K. Welker, Structured inverse modeling in parabolic equations, arXiv: $1506.02244 \mathrm{v} 4$ [math. OC] 5 Jul. 2015.

[19] V. Schulz, Martin Siebenborn, and Kathrin Welker, Towards a lagrange-newton approach for pde contrained shape optimization, arxiv: 1405. 326v2 [math.NA] 27 nove 2014.

[20] L. Silvestre, On the differentiability of the solution to an equation with drift and fractional diffusion, Indiana University Mathematical Journal. 61 (2002), no. 2, 557584. 11, 16, 39.

[21] L. Silvestre, Regularity of the obstable problem for a fractional power of the Laplace operator, Comm. Pure Appl. Math. 60 (2007), 67-112. 1, 9, 15.

[22] E. Val , D. Chamorro, Modelisation de l'operateur Laplacien Fractionnaire: A travers un probléme d'extension au demi-espace, 2014.

[23] M. Warmi, The fractionnal Relative Capacity and the Fractionnal laplacian with Neumann and Robin Boundary conditions on open sets, (X), 2014. 